\title{
Connecting the Dots: Children's Use of a Systematic Figure to Facilitate Mapping and Search
}

\author{
David H. Uttal, Vanessa H. Gregg, Lisa S. Tan, Meghan H. Chamberlin, and Amy Sines \\ Northwestern University
}

\begin{abstract}
Organizing locations into a systematic figure was predicted to facilitate children's use of spatial relations in a mapping task. In Study 1,3-, 4-, and 5-year-olds used a map to find a sticker hidden under 1 of 27 locations. The search locations formed a systematic figure, the outline of a dog. Half of the children were shown that the locations formed a dog. Seeing the dog pattern facilitated the performance of 5-year-olds but not that of the younger children. Study 2 indicated that children had to see a systematic figure to gain an advantage; adding lines to an unsystematic figure did not convey an advantage. Study 3 indicated that a verbal label alone could not convey an advantage. Study 4 revealed that seeing the dog pattern could also facilitate performance when the map was rotated relative to the represented space. The importance of organizing spatial information to facilitate relational thinking and mapping is discussed.
\end{abstract}

The ability to understand maps and models is critical to communication and travel. Maps free us from the bounds of our own direct experience. They allow us to see, and think about, substantially more spatial information than we could ever experience directly. Our conceptions of the world therefore are influenced by the representation of spatial information on maps. Coming to think about the relation between maps and the spaces that they represent is an important aspect of the development of spatial cognition (Liben, 1999; Uttal, 2000; Wood, 1992).

Children understand some aspects of maps and models at an early age. For example, at age 3 , children can use a simple map or model to find a hidden toy, and by age 4 they can follow mapdepicted routes (Blades \& Spencer, 1987; Bremner \& Andreasen, 1998; DeLoache, 1987, 1989, 2000; Dow \& Pick, 1992; Landau, 1986; Marzolf \& DeLoache, 1994; Uttal \& Wellman, 1989). However, this early competence is not the end of the developmental story (Liben, 1999). In most previous studies, young children performed well only on tasks that could be solved on the basis of object correspondences. An object correspondence involves a one-

David H. Uttal, Vanessa H. Gregg, Lisa S. Tan, Meghan H. Chamberlin, and Amy Sines, Department of Psychology, Northwestern University.

Portions of this work were presented at the 1996 Conference on Human Development, Birmingham, Alabama, and at the 1997 meetings of the Association of American Geographers, Fort Worth, Texas. Portions of the work also appeared in an honors thesis submitted by Vanessa H. Gregg to the Department of Psychology at Northwestern University.

The work was supported by Grants R01HD25271 and R29HD34929 from the National Institute of Child Health and Human Development and Learning in Intelligent Systems Grant NSF 97201313 from the National Science Foundation.

We are especially grateful for the contributions of Judy DeLoache and Dedre Gentner throughout all stages of the work. Thanks also to Karen Bartsch, Dahlia Hassani, Maeve Hennerty, Linda Liu, Lauren Mather, Beth Proffit, and Bill Stein for their comments and suggestions.

Correspondence concerning this article should be addressed to David H. Uttal, Department of Psychology, Northwestern University, 2029 Sheridan Road, Evanston, Illinois 60208-2710. Electronic mail may be sent to duttal@northwestern.edu. to-one relation between a single location on the map (or in the model) and the corresponding location in the represented space (Blades \& Cooke, 1994; Liben, 1999; Loewenstein \& Gentner, in press). For example, in DeLoache's (1987) original studies, the locations in the model and in the referent space were individual, unique furniture items. There was a single couch in the room and a corresponding miniature couch in the model. Likewise, there was a single chair in the room and a corresponding miniature chair in the model. Many researchers (e.g., Bence \& Presson, 1997; Blades \& Cooke, 1994; Blades \& Spencer, 1994; Lillard, 1993; Loewenstein \& Gentner, in press; Marzolf, DeLoache, \& Kolstad, 1999; Perner, 1991) have suggested that children can solve objectcorrespondence mapping tasks simply by noticing the relation between the depiction of single locations on the map and the corresponding locations in the space. They do not need to think about the spatial relations among the locations. For example, they need to note only that the toy is behind the (single) couch; they do not need to take into account that the couch is to the left of the chair.

Young children perform much worse on tasks that require the use of spatial correspondences, which are mappings that do take spatial position into account. Spatial correspondences must be used when some or all of the hiding locations are not unique. For example, Blades and Cooke (1994) asked children to find a toy that was hidden under a table, a couch, or one of two identical chairs that were placed in different positions. Children were shown where the toy was hidden in one model, and they were then asked to find the toy in the corresponding location in a second, identical model. Four-year-olds performed well when the toy was hidden behind the table or the couch. However, the same children performed at chance levels $(50 \%)$ when the toy was hidden behind or under one of the two chairs. The children knew to search behind a chair, but they did not know which chair. Similarly, Liben and Yekel (1996) found that 4- and 5-year-olds had trouble placing stickers on a map to indicate classroom locations when the correct position could only be differentiated on the basis of spatial position.

The focus of this article is on the development of children's use of spatial correspondences. We suggest that the challenge of using 
spatial correspondences can be construed in terms of the development of the ability to understand and use other kinds of relations, such as those involved in making an analogy. Previous work has shown that young children often interpret relations primarily on the basis of superficial, physical similarities between the two objects or sets of knowledge. For example, if 5-year-olds are asked "How are clouds and sponges alike?" they often say that both are white and fluffy. In contrast, 9-year-olds are more likely to say that both hold and later release water (Gentner \& Ratterman, 1991). Thus, the development of analogical reasoning consists partly of a relational shift, in which children gradually come to focus less on object correspondences and more on relational correspondences (Gentner \& Ratterman, 1991; Gentner \& Toupin, 1986; Kotovsky \& Gentner, 1996; Loewenstein \& Genter, in press; see also Schoenfeld \& Herrman, 1982; Silver, 1981).

Although young children sometimes have trouble reasoning about relations, their performance can be enhanced substantially by several techniques that highlight relations or make them more tractable (Gentner \& Ratterman, 1991). For example, Gentner (1977) asked young children to solve simple spatial analogies such as "If a tree had a knee, where would it be'Tis task required that children complete an analogy on the basis of relations. The children first needed to think about where a knee was located in relation to the body. Then they had to map this relation to a tree, and finally, they had to use this mapped relation to infer the spatial position of the "knee" of the tree. Despite these challenges, 4- and 5 -year-olds performed well even when the tree was shown in a noncanonical orientation, such as lying on the ground. They could use their well-honed knowledge of the relations among parts of the human body as a basis for reasoning about an inferred relation, the knee of a tree. In sum, previous work on analogical reasoning has revealed (a) that young children often have difficulty reasoning about relations and (b) that their performance can be improved substantially by providing information that facilitates reasoning about relational information.

We suggest that thinking of the use of maps as a form of analogy can shed new light on the study of the development of spatial cognition and map-reading skills (Loewenstein, 2000; Loewenstein \& Gentner, in press; Uttal, Loewenstein, \& Gentner, 2000). To use a map effectively, the map reader must establish connections (mappings) between sets of locations on the map and in the corresponding space. These mappings can be made using either superficial object correspondences or more relational spatial correspondences. The difficulty that children experience in using spatial correspondences is similar to the general difficulty that they have in using other kinds of relational information. The child must think, in essence, that the set of relations on the map is like the corresponding set in the space that the map represents. Thinking about sets of relations is precisely the kind of task that typically is difficult for young children.

On the basis of work in analogical reasoning, we predicted that children's use of spatial correspondences would be enhanced if we made the (spatial) relational information more tractable and easier to use. Specifically, we investigated the influence of one facilitative factor, systematicity, that may be particularly relevant to the use of spatial correspondences. As used here, the term systematicity refers to a hierarchical structure in which individual locations or bits of information are related in an ordered and predictable fashion to each other and to the entire structure or body of knowledge when considered as a whole (Gentner \& Toupin, 1986; Palmer, 1975). Embedding elements of a problem within a systematic structure facilitates analogical reasoning substantially. For example, consider the analogy "The atom is like the solar system." This analogy is comprehensible largely because of the systematic relation between the planets and the sun. The planets and the sun are systematically related because (a) each planet shares the same type of relation with the sun-an orbit, and (b) the orbits of the planets are hierarchically related to each other. Thus the planets and the sun form a coherent and predictable system of relations. The systematicity of the planet-sun relations leads people to look for corresponding sets of relations in the atom, and thus they can solve the analogy. Note that providing information about particular objects (and not relations among these objects) does not help people perform better. For example, pointing out that Mars is red or that Jupiter is large does not help people to comprehend the relations among the solar system and the atom.

Systematicity may be particularly relevant to the study of spatial cognition and the development of map use. Indeed, since the time of the Gestaltists, psychologists have stressed that the perception and interpretation of spatial information is not merely a process of keeping track of individual locations (Anderson, 1995; Koffka, 1963; Kohler, 1947; Palmer, 1975). Instead, people often interpret individual locations as part of an organized or systematic figure. Well-known figures such as the outlines of animals or people often are used as a framework for organizing spatial locations. For example, it is not a coincidence that many of the constellations are based on animate figures. These figures are systematic because the individual parts can be embedded easily within an organized structure that highlights the relations among the parts. For example, the legs and arms are related to each other and to the body in a well-known, hierarchically organized, and predictable fashion. Knowledge of these relations can facilitate the use of maps or charts of the stars. People can search in the evening sky for a well-known pattern that they saw on the map even though the actual structure (e.g., the dipper) is not visible (see also Hunt \& Agnoli, 1991). What matters most is that we know to look for a set of relations among the individual elements.

In the present work we investigated whether embedding a set of locations within a systematic pattern would facilitate children's use of spatial correspondences. In doing so, we sought to provide evidence for the general claim that using a map can be considered a form of analogy and that children's difficulty in using spatial correspondences is a form of the general difficulty that they experience in thinking about relational correspondences. We focused on children ages 3 through 5 years because we hoped to bracket the age at which the ability to take advantage of the systematic pattern might emerge. Between the ages of 3 and 5 years, children develop the ability to understand object correspondences, but they still struggle in using spatial correspondences (see Bence \& Presson, 1997; DeLoache, 1989; Liben \& Downs, 1989, 1993; Liben \& Yekel, 1996).

We asked children to use a map to find a sticker that was hidden among 1 of 27 locations that were identical except for spatial position. We chose a large number of nonunique locations to ensure that the task required that children use spatial correspondences to find the stickers. The locations were distributed across the space in a pattern that could be interpreted as the outline of a dog. We showed half of the children a map on which the locations 
were connected with lines to highlight the dog pattern. The remaining children used a map without lines; these children did not see the outline of the dog. We predicted that seeing the lines would facilitate children's performance. When the locations were embedded within the dog pattern, their spatial relations were no longer arbitrary; they became related to each other in a systematic, predictable way. Children could use this information in two related ways. First, they could use the dog pattern to help them remember the locations on the map. The locations could be encoded relative to the well-known structure of the dog's body. Second, the children could use the dog pattern to facilitate mapping of spatial information. The dog pattern could provide a structure that could be mapped to the otherwise unrelated locations in the space. This would help the children establish connections between what they saw on the map and what they saw in the room.

We report the results of four studies. Study 1 demonstrated that seeing the dog pattern on the map improved 5-year-olds' performance substantially. Studies 2 and $\mathbf{3}$ helped to clarify the source of the effect observed in Study 1. Study 4 demonstrated that seeing the dog pattern could also facilitate performance in a much more difficult task in which the map and the space were misaligned.

\section{Study 1}

\section{Method}

Participants. The participants were 25 three-year-olds (mean age $=$ 41.3 months, range $=36.2-46.9$ months), 24 four-year-olds (mean age $=51.8$ months, range $=48.6-53.8$ months), and 24 five-year-olds (mean age $=63.4$ months, range $=60.3-65.8$ months) approximately equally divided between the two sexes. The children were recruited through direct mail to their parents.

Materials. The search space was a $10 \times 10 \mathrm{ft}(3 \times 3 \mathrm{~m})$ yellow felt carpet that was placed within a larger room. There were windows on two of the four walls of the larger room, but the windows were not shown on the maps. The search locations were 27 blue paper coasters (radius $=10$ $\mathrm{cm}$ ) that were distributed across the felt carpet in a pattern that formed the shape of a dog, as shown in Figure 1. Children searched for small stickers and collected them in a small bucket.

Figure 1 also shows the two maps, which we drew on $1 \mathrm{X} 1 \mathrm{ft}$ $(30.5 \times 30.5 \mathrm{~cm})$ sheets of yellow cardboard. Small circles (radius $=1 \mathrm{~cm})$ were drawn on the maps in blue ink to represent the locations of the coasters on the felt carpet. On the lines map, we drew lines between the circles to form the dog pattern. The no-lines map showed only the circles.

Procedure. Half of the children in each age group (the lines group) were assigned randomly to use the lines map; the remaining children (the no-lines group) used the no-lines map.

The experimenter first explained the task, giving identical instructions to the two groups. The goal of the explanation was to familiarize the child with the task and the relevance of the map for finding the stickers. The experimenter did not say or otherwise indicate that the locations could be construed as forming the outline of a dog. The experimenter showed the participant the felt carpet and the map for the condition to which he or she was assigned. Next, the experimenter pointed out the correspondences between a few locations on the map and those in the space (see Figure 2). At the same time, the experimenter explained the task. While pointing first to the map and then to the corresponding locations, the experimenter said the following:

These two dots here are these two dots right here. [Name of assistant] is going to hide stickers underneath the dots on the carpet, and I'm going to show you where she [or he] hides them on the map. Ok? And you're going to use the map to find the stickers - and you get to keep all the stickers you find! So the first thing we're going to do is go behind here and wait here while [name of assistant] hides the sticker so we can't see where she [or he] hides it.

The child was then given two practice trials during which the sticker was hidden at the locations shown in Figure 2. The experimenter showed the child a location on the map and asked, "Can you go to that dot on the carpet and pick it up?" The child was allowed to search three times on each trial. If he or she had still not found the sticker, the experimenter lifted the correct coaster.

The experimenter then began the test trials. She asked the child to go with her behind the partition, to the location represented by the circle in Figure 2. The experimenter said, "Now we're going to keep the map back here, so you're going to have to remember where I point on the map." An assistant simultaneously hid a sticker in the room, out of sight of the child. The experimenter indicated the circle on the map that corresponded to the coaster under which the assistant was hiding the sticker in the room. The experimenter asked the child to point to the map location three times to ensure that he or she remembered the correct location.

The child and the experimenter then walked around the partition, leaving the map behind. The experimenter asked the child to turn over the coaster under which the sticker was hidden. As in the orientation trials, the child was given three chances on each trial to find the sticker, after which the experimenter picked up the correct coaster. On each of the 10 trials, the sticker was hidden at one of the locations indicated in the top panel of Figure 1. There were four different, randomly selected orders of search locations.

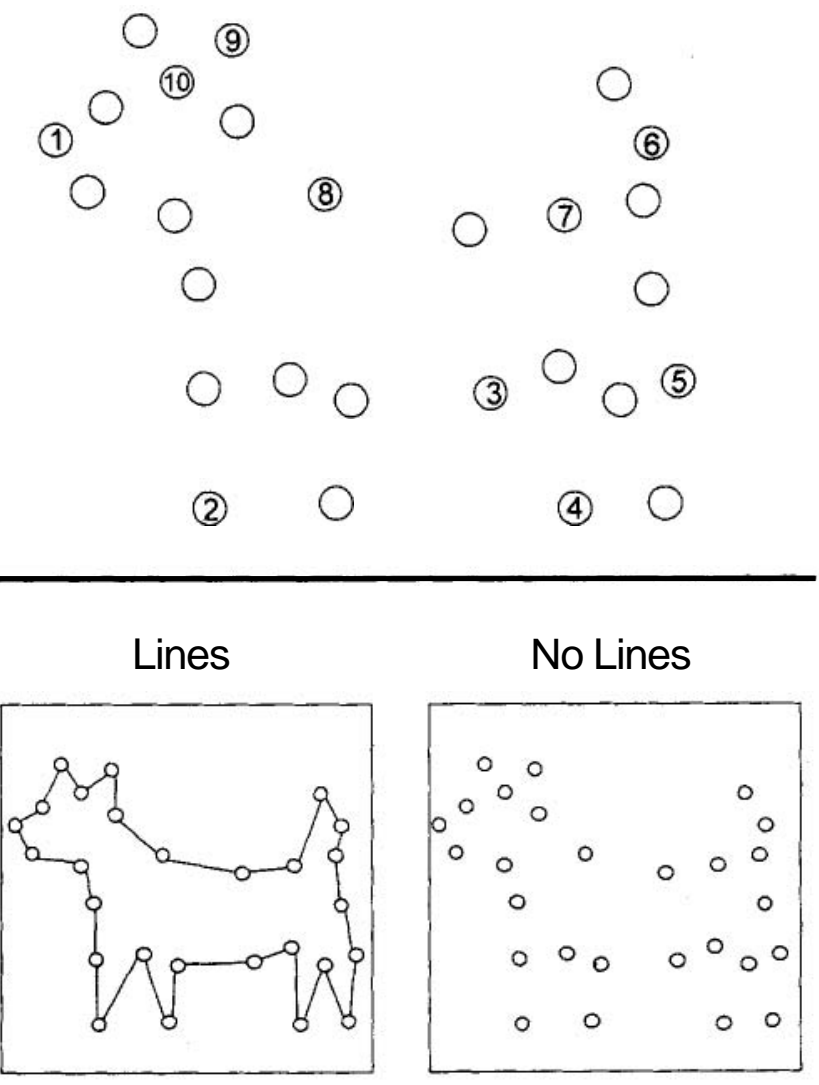

Figure I. The configuration of search locations (top panel) and the two maps (bottom panel) used in Study 1. The numbers in the top panel represent the 10 hiding locations. 

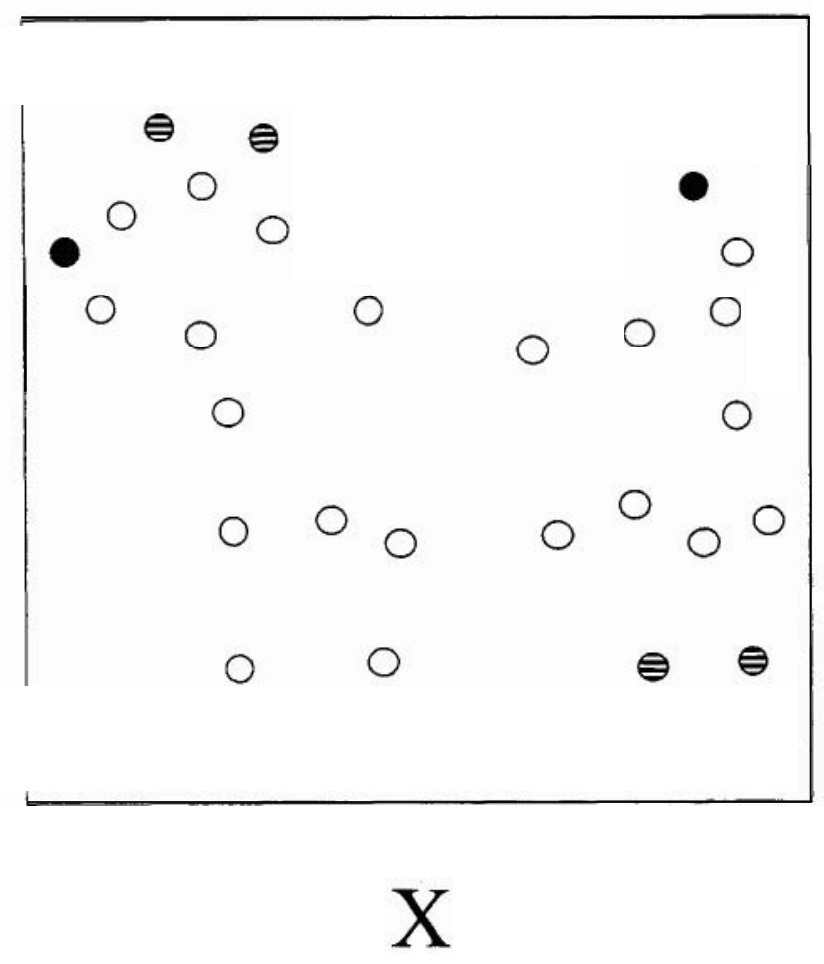

Figure 2. The layout of the testing room and the demonstration locations. The solid line represents the opaque barrier. The partly darkened circles represent the locations to which the experimenter pointed during the initial explanation, and the fully darkened circles represent the locations at which the sticker was hidden during the practice trials. During the explanation, the child and experimenter stood at the location marked with the X. During the test trials, the experimenter showed the child the map while sitting at the location marked with the $\mathbf{O}$. (The figure is not drawn to scale.)

\section{Results}

Defining correct performance. In the analyses that we report we used a conservative definition of correct performance: A search was scored as correct only if the child's first choice was the correct coaster. However, we also conducted all of the analyses using a more liberal criterion: A search was scored as correct if the child found the sticker on any one of the three attempts that the child was allowed on each trial. In almost all cases, using the more liberal criterion affected only the magnitude of performance; it did not affect the pattern of differences among conditions, age groups, or the sexes. Consequently, only the conservative-criterion results are reported. The main dependent variable was the total number of correct searches across the 10 trials.

Overall performance. Figure 3 shows children's performance as a function of age and condition. A 3 (age) by 2 (condition) by 2 (sex) analysis of variance (ANOVA) revealed a significant interaction between age and condition, $\mathrm{F}(2,61)=3.5, p<.05$. Simple effects tests revealed that seeing the lines on the map did not affect the performance of the 3- and 4-year-olds. However, the 5-yearolds in the lines group $(\mathrm{M}=7.1, S D=1.6)$ performed significantly better than the 5-year-olds in the no-lines group $(\mathrm{M}=\mathbf{5 . 4}$, $S D=1.3) . \mathrm{F}(1,61)=4.7, \mathrm{p}<.05$.

The analysis of the performance of individual children was consistent with the analysis of the overall levels of performance. For this analysis, we defined successful performance as the mean level of performance of the 5-year-olds in the lines group (i.e., $70 \%$ correct searches). Seven of the 12 five-year-olds in the lines group, but only 2 of the 12 five-year-olds in the no-lines group, performed this well, $\mathrm{X} 2(1, \mathrm{~N}=24)=4.4, \boldsymbol{p}<.05$. Similar analyses were also conducted for the other age groups, but no significant differences emerged.

Comparison with chance performance. The results presented thus far indicate that seeing the dog pattern facilitated only the 5-year-olds' performance. One possible explanation of this result is that the younger children failed to use the map at all. In this view, the children would have searched randomly in the room regardless of what they saw on the map. If children were searching randomly, then we would not expect that seeing the lines on the map would affect their performance. For these reasons, we conducted additional analyses to determine if children performed better than chance.

There were 27 locations, and children could search at any location on each of the 10 trials. We therefore assumed that chance performance would be 10127 , or .37 . We compared the performance of all groups of children to this level with one-sample $t$ tests. All groups performed significantly better than chance except the 3-year-olds in the lines group, ts $(11-12)>3.6$, ps $<.01$. Thus, most of the 3- and 4-year-olds performed better than chance, but seeing the dog pattern did not help them perform better.

Error analysis. The analysis of children's errors shed light on how seeing the dog pattern helped the 5-year-olds in the lines group. In general, when 5-year-olds in either group made errors, they still searched very close to the correct locations. A search was defined as a proximate error if a child chose an incorrect location that was one of the three closest to the correct location. Two thirds of the 5-year-olds' errors $(\mathrm{M}=66.1 \%, S D=30.4 \%)$, but

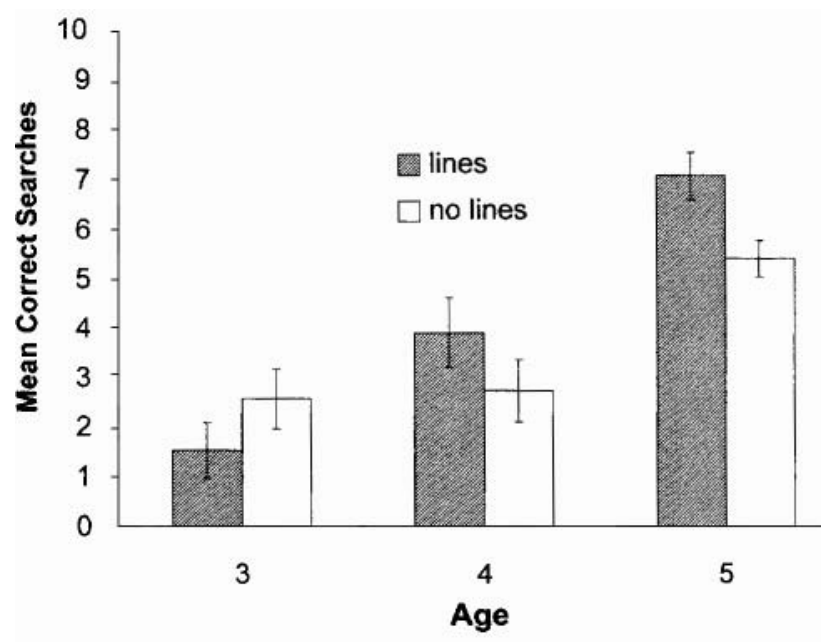

Figure 3. Average number of correct searches (out of 10) for 3-, 4-, and 5 -year-olds in the lines and no-lines groups. 
only $29.9 \%(\mathrm{SD}=22.1 \%)$ of the 4 -year-olds' errors and $15.7 \%$ $(\mathrm{SD}=18.3 \%)$ of the 3-year-olds' errors, were proximate errors. A 2 (age) by 2 (condition) by 2 (sex) ANOVA on the proportion of proximate errors showed only a main effect of age, $\mathrm{F}(1$, $61)=26.4, \mathrm{p}<.001$.

Although both groups of 5-year-olds searched close to the correct locations, there were still important differences in their error patterns. These differences may reflect how children were searching for the sticker. In this series of analyses, we focused only on the 5-year-olds because seeing the dog figure did not affect the performance of the 3- or 4-year-olds. Figure 4 shows the pattern of searches at the three locations at which the performance of the two groups of 5-year-olds differed the most. The figure reveals that the no-lines group often searched incorrectly at locations that could be construed as lying roughly in line with the target locations. Consider, for example, the target location shown in the top panel in Figure 4. Nine of the 12 children in the lines group, but only 3 of the 12 in the no-lines group, searched correctly at this target location. Five of the 9 incorrect searches of the no-lines group were at locations immediately to the left or right of the target configuration. Similarly, the 5-year-olds in the no-lines group had difficulty avoiding the foil locations to the right and left of the target location shown in the middle panel of Figure 4.
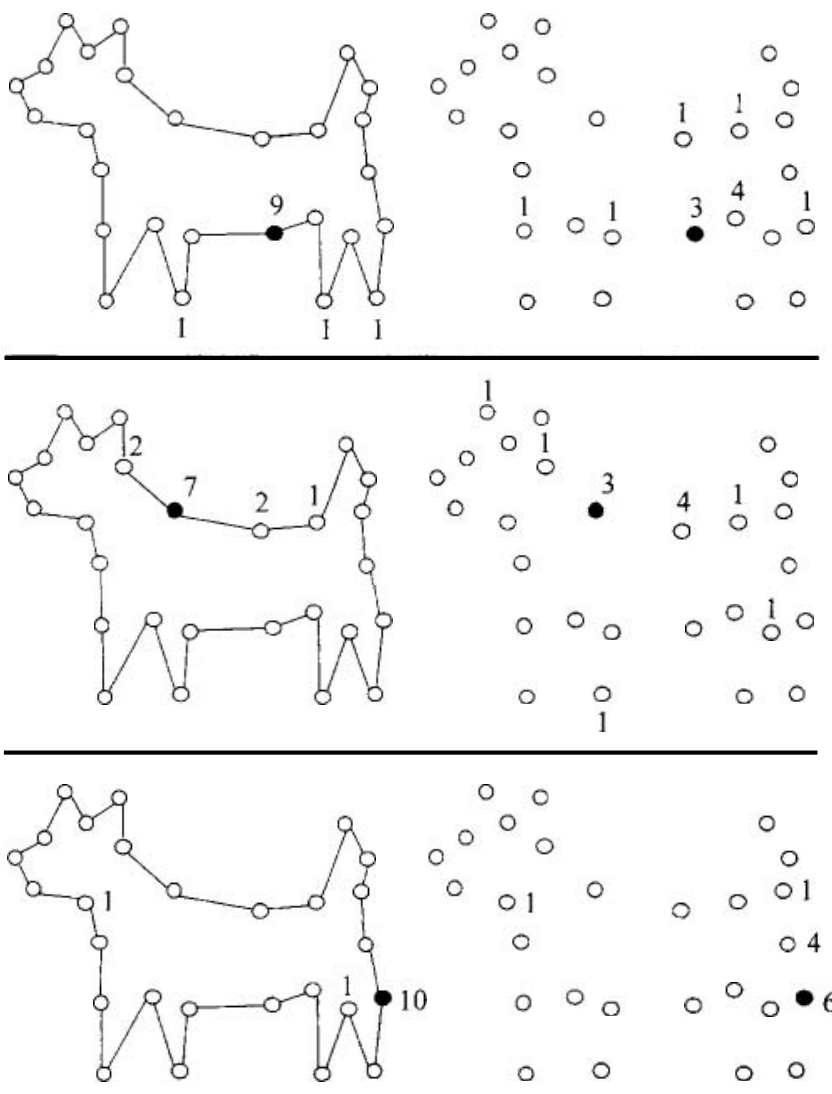

Figure 4. Patterns of searches for the three locations at which the performance of the 5-year-olds in the lines and no-lines groups differed the most. The darkened circles represent where the sticker was hidden. The numbers indicate how many children (out of 12) searched at a given location.
The pattern of searches suggests that children in the lines group were able to take advantage of the dog pattern to avoid searching at these difficult foil locations. For example, the locations both to the right and to the left of the target in the top panel of Figure 4 are in different parts (i.e., legs) of the dog. Thus, seeing the dog figure may have allowed the children to go beyond processing the information solely on the basis of left-right (or up-down) relations. Instead, they could rely on the dog figure to avoid searching at the foils that the 5-year-olds in the no-lines group found difficult to avoid.

\section{Discussion}

The results indicate that thinking of a set of locations in terms of a systematic figure can facilitate children's mapping and search skills substantially. In interpreting this finding, it is important to note that the lines that we used to illustrate the dog pattern were present only on the map. There were no lines in the actual search space; children saw only the 27 coasters scattered across the carpet. Consequently, the results cannot be attributable solely to matching a pattern of lines on the map to a similar pattern in the space that the maps represented.

Instead, the results suggest that seeing the dog pattern altered how children thought about the locations and searched among them. The 5-year-olds in the no-lines group performed poorly when searching at locations that were spatially difficult (i.e., collinear) to discriminate from other nearby locations. To these children, the target and nearby locations may have appeared as a set of coasters roughly in line with each other. Thus the no-lines group often had to discriminate the correct location from nearby foils that were to the left or right of the target. This is a difficult task for young children (Corballis \& Beale, 1976; Olson \& Bialystock, 1983). In contrast, the 5-year-olds in the lines group were able to reject the locations that were in line with the target locations. They could use the systematic dog pattern to augment their knowledge of the location of the stickers. The results suggest that the 5-year-olds who saw the dog pattern may have been able to avoid the difficult foil locations by realizing that the foils were in different parts of the dog than the target location was (see Figure 4). The systematic pattern that they saw on the map made it possible for them to see that the locations were related to each other in a predictable, orderly way. After the presentation of additional research, we consider in more detail how seeing the dog pattern facilitated performance.

In sum, the results of Study 1 indicate that becoming aware of the dog pattern facilitated 5-year-olds' performance substantially. In Studies 2 and 3 we investigated in more detail the cause of this advantage.

\section{Study 2}

Our goal in Study 2 was to isolate the causes of the effects observed in Study 1. We focused on the consequences of adding lines to the configuration of locations. Recall that in Study 1, we showed children the dog figure by connecting the locations on the map with lines. Consequently, the lines map differed from the no-lines map in two ways (see Figure 1): (a) the presence of lines per se and (b) that the lines formed the outline of a dog. 
The superior performance of the 5-year-olds who knew about the dog pattern in Study 1 could conceivably have been due to either of the differences between the lines and no-lines maps. It is possible that connecting the locations with lines would be enough to facilitate children's performance regardless of whether the lines formed a systematic pattern. In this view, the superior performance of the 5-year-olds in the lines group in Study 1 could have been due, at least hypothetically, to a division of the figure into parts or regions based purely on geometric features, such as local minima (Biederman, 1987; Boltyanskii \& Gohberg, 1980; Hoffman \& Richards, 1984; Hoffman \& Singh, 1997; Tam \& Pinker, 1989; Tversky, 1989; Tversky \& Hemenway, 1984). Thus, it would not matter that the lines revealed a systematic figure; all that would matter was that the lines formed local, unrelated geometric parts.

In Study 2, we investigated whether the effect that we observed in Study 1 depended on the children's seeing a systematic figure. We asked 5-year-olds to search among a set of locations that was similar to the dog pattern in terms of spatially defined parts but whose parts did not form a systematic figure. As in the previous studies, half of the children used a map on which the individual locations were connected with lines; the remaining children used a map without lines. The critical question was whether adding lines to the new, unsystematic configuration would facilitate children's performance. A negative finding would suggest that the superior performance of the 5-year-olds in the lines group of Study 1 was at least partly due to the systematic arrangement of the parts of the dog figure.

\section{Method}

Participants. There were 24 five-year-olds (mean age $=62.2$ months, range $=60-65$ months) approximately equally divided between the two sexes. None of the children from Study 1 participated in Study 2

Materials. The materials were identical to those used in Study I except that the pattern of locations was different. Figure 5 shows the configuration and the lines and no-lines maps. We created the new figure by rearranging the parts of the dog.

Procedure. The instructions and procedures were the same as those of Study 1.

\section{Results and Discussion}

The lines $(\mathrm{M}=4.2, \mathrm{SD}=2.1)$ and no-lines $(M=4.5$, $\mathrm{SD}=2.1$ ) groups performed almost identically. A 2 (lines) by 2 (sex) ANOVA revealed no significant effects or interaction. This result contrasts substantially with the results of Study 1, in which the 5-year-olds in the lines group performed almost 30\% better than the 5-year-olds in the no-lines group. An analysis of the children's errors revealed no significant or consistent differences between the two groups. The performance of both groups in Study 2 was comparable to that of the no-lines group of Study 1, $\mathrm{F}(2,30)=1.1$, ns. This result suggests that the advantage associated with using the lines map in Study 1 was that it helped children to see that the locations formed the outline of a systematic figure, the dog. Any other explanation would need to account for why the particular arrangement of geometrically defined parts that we used in Study 1 conveyed a large advantage whereas the arrangement that we used in Study 2 conveyed no advantage.

In sum, the results of Study 2 highlight the unique importance of the systematic arrangement of the parts of the dog figure. The

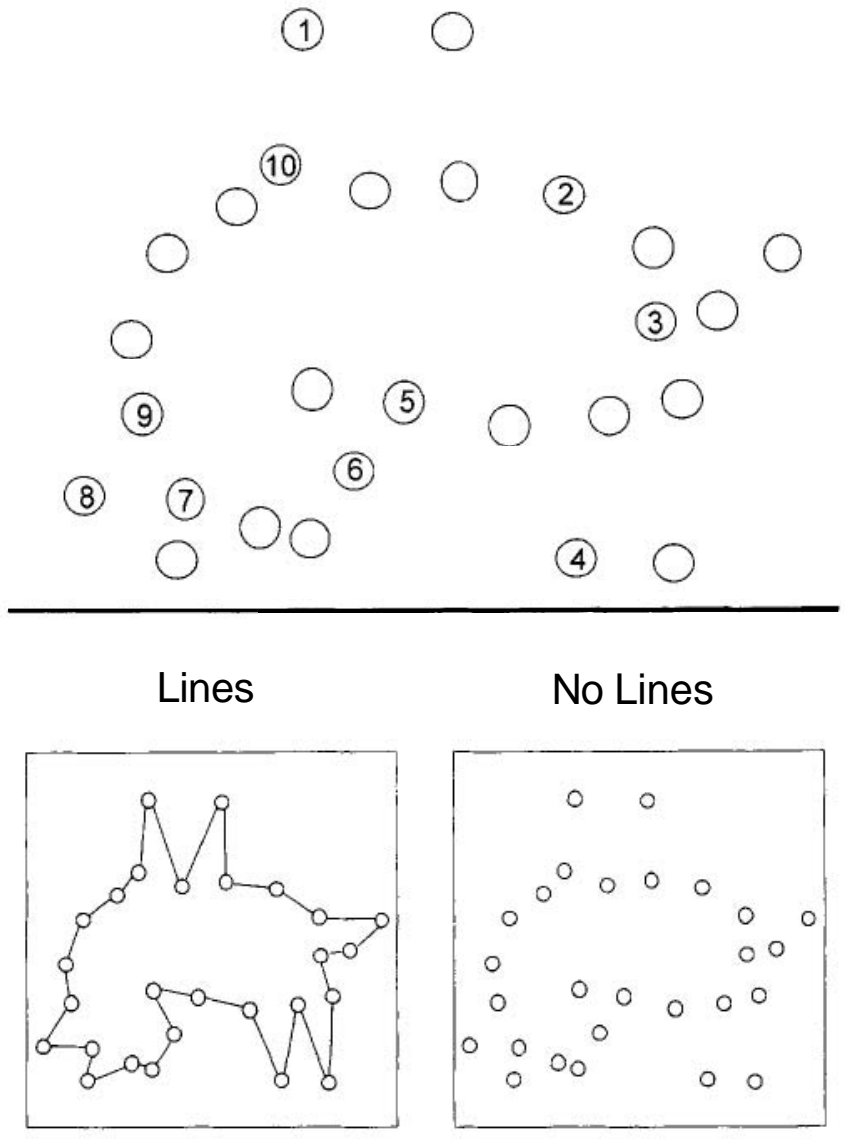

Figure 5. The configuration of search locations (top panel) and the two maps (bottom panel) used in Study 2. The numbered locations represent 10 hiding locations.

Study 2 figure had the same parts as the Study 1 figure, but these parts were not arranged to form a systematic pattern. Consequently, the addition of lines to the Study 2 figure did not facilitate children's performance. The results suggest that the children could not use parts of a figure unless these parts were related systematically to a recognizable whole, the dog (see Gentner \& Toupin, 1986; Morrison \& Tversky, 1997; Stiles, Delis, \& Tada, 1991; Tversky, 1989, 1997). Systematicity helps people to think about relations, and consequently children did not benefit from seeing parts that were not related to each other.

\section{Study 3}

The results of Study 2 indicated that the effect of seeing the dog pattern was not attributable solely to the addition of lines per se, but rather to what the lines formed. This finding raises the question of whether children must actually see the dog or whether we could communicate in a different way that the locations could form the outline of a dog. Perhaps hearing that the locations could form the outline of a dog would be sufficient. The notion that a label might be enough to facilitate children's performance is consistent with the results of some prior studies that have shown that labeling can facilitate children's encoding, recall, and reconstruction of spatial information (e.g., Stiles, Tada, \& Whipple, 1990). 
In Study 3, we investigated whether providing a label for the configuration of locations would convey an advantage in the search task. As in Study 1, half of the participants used the lines map, and the remainder used the no-lines map. We told all of the children that the locations formed the outline of a dog. Comparing the results of Study 3 with those of Study 1 therefore allowed us to address two questions: whether a label alone could facilitate performance and whether a label could lead to even better performance in children who did see the lines on the map.

\section{Method}

Participants. There were 24 five-year-olds approximately equally divided between the two sexes. The children were recruited from the same sources used in Study 1.

Procedure. The procedure was identical to that of Study I with one exception: During the orientation phase, the experimenter also said, "If you look at all these dots here, you can see a dog. See, here's the head and here's the tail." The experimenter pointed to locations in the appropriate areas while providing these labels. Thereafter, the procedures were identical to those of the previous studies.

\section{Results and Discussion}

As in Study 1, the lines group $(\mathrm{M}=7.6, \mathrm{SD}=1.5)$ performed significantly better than the no-lines group $(\mathrm{M}=5.6, \mathrm{SD}=2.4)$, $\mathrm{F}(1,20)=5.8 \mathrm{p}<.05$. The effect of sex was not significant.

To assess the effect of the label on children's searches, we compared the results of Study 3 with those of the 5-year-olds from Study 1 . In combination, the two studies form a 2 (lines) by 2 (label) by 2 (sex) design. An ANOVA revealed only the previously reported main effect of lines; in both studies, the lines groups performed better than the no-lines groups. The label did not affect children's performance. Children who heard the label $(\mathrm{M}=6.6$, $\mathrm{SD}=2.2$ ) performed about the same as those who did not hear a label $(\mathrm{M}=6.3, \mathrm{SD}=1.7), \mathrm{F}(1,40)<1$, ns.

The results may indicate that children need to see the dog structure to gain an advantage. The label told children only that the locations could form the outline of the dog; children would still need to figure out how the locations could be embedded within the dog pattern. Five-year-olds may have found it difficult to figure out on their own how the locations could form a dog. This conclusion would be consistent with the results of previous work (e.g., Vurpillot, 1976) that suggested that 5-year-olds may be less likely than older children to interpret locations in terms of wellknown patterns. However, further studies would be required before one could conclude that 5-year-olds could not benefit from hearing the label. For example, children might benefit from hearing the label on every trial or from a more detailed explanation of how the locations could form a dog. In sum, the results of Study 3 indicate that the effects we observed in Study 1 are replicable and that hearing a verbal description of the configuration of locations does not affect children's performance.

\section{Study 4}

The results presented thus far demonstrate a specific advantage of using the dog figure to facilitate search. In Study 4, we extended the findings by showing that the figure could also facilitate children's performance in a much more difficult task. We rotated the map either $+90^{\circ}$ or $-90^{\prime \prime}$ relative to the represented space. Typically, young children (and sometimes even adults) have great difficulty using rotated maps or configurations (Blades, Sowden, \& Spencer, 1995; Bluestein \& Acredolo, 1979; Levine, Jankovic, \& Palij, 1982; Liben \& Yekel, 1996; Presson, 1982; Pufall \& Shaw, 1973). We predicted, however, that seeing the dog pattern might alter how children interpreted and mapped the relations between locations on the map and in the space. Consequently, children who saw the map with lines might perform relatively well in our task even when the map was not aligned with the search space.

This prediction is derived from a review of the kinds of errors children made in previous studies. Typically, young children failed to compensate for the rotation of the map. They behaved instead as if the map and space were properly aligned (Blades et al., 1995; Bluestein \& Acredolo, 1979; Presson, 1982; see also Huttenlocher \& Presson, 1973, 1979; Pufall \& Shaw, 1973). Their searches often were off target by the degree of rotation. Figure 6 provides a hypothetical example that is relevant to the present research. In this example, the sticker was hidden at a location in the face of the $\mathrm{dog}$, and the map was rotated 90" to the left. On the rotated map, the face section of the dog appeared relatively close to and in front of where children sat when they viewed the map. Children who failed to compensate for the rotation would search at one of the locations closest to where they stood in the space before searching for the sticker. These failure-to-compensate errors are particularly common in rotated-maps tasks in which there are no clear landmarks that can be used to establish relations between the map and the space (Blades et al., 1995; Presson, 1982).

We hypothesized that seeing the systematic dog pattern might help children overcome the difficulty that they typically have in compensating for the rotation of the map. In this case, the specific advantage would be that the relations among locations would remain constant after the rotation. Children who saw the dog pattern might therefore be able to establish correspondences on the basis of the systematic and rotationally invariant relations among parts of the dog. They would not need to rely (as much) on the assumed correspondences between the individual locations on the map and in the space. Consequently, we predicted that the lines group would perform better than the no-lines group. A corollary
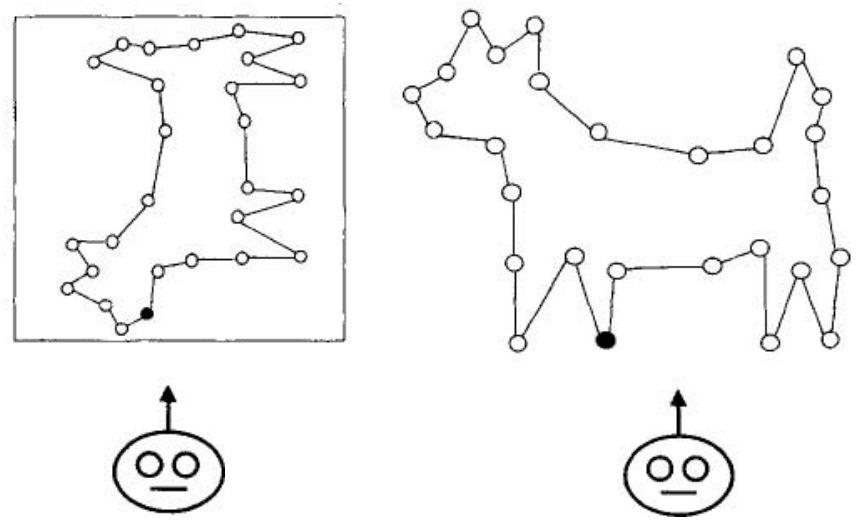

Figure 6. An example of a failure-to-compensate error. The faces represent the perspective from which the children viewed the maps and the orientation in which they stood before searching in the room. 
prediction was that children in the lines group would commit relatively few failure-to-compensate errors.

\section{Method}

Participants. The children were $\mathbf{2 4}$ five-year-olds (mean age $=\mathbf{6 2 . 9}$ months, range $=\mathbf{6 0 . 1}-65.9$ months) approximately equally divided between the two sexes. None of the children had participated in the prior studies.

Materials. The materials were the same as those used in Study 1.

Procedure. The procedure was identical to that of the previous studies except that we rotated the map $\mathbf{+ 9 0}^{\circ}$ (for half the children) or $\mathbf{- 9 0}^{\prime}$ (for the remainder of the children). Half of the children saw the lines map, and the remainder saw the no-lines map. During the initial explanation, the map was not rotated. Thus the children saw correspondences between the map and the space when the two were correctly aligned. After pointing out these correspondences, the experimenter rotated the map and said, "Now we're going to turn the map this way. You will have to remember that we turned the map." The map remained in the rotated orientation for the remainder of the experiment.

\section{Results}

As predicted, the lines group $(\mathrm{M}=4.2, \boldsymbol{S D}=2.4)$ performed significantly better than the no-lines group $(\mathrm{M}=0.5, S D=0.9)$, $F(1,20)=27.7, p<.001$. The direction of rotation did not affect the accuracy of children's performance.

Additional analyses addressed whether children made failureto-compensate errors (see Figure 6). To define failure-tocompensate errors, we rotated the entire configuration $+90^{\circ}$ and $-90^{\prime \prime}$ and calculated the rotated coordinates of all 27 search locations (see Smart, 1986; Uttal, 1996). We defined a search as a failure-to-compensate error if the child picked up one of the five coasters that was closest to the rotated coordinates of the target location.

As shown in Figure 7, the no-lines group, $(\mathrm{M}=4.5, S D=2.1)$ committed many more failure-to-compensate errors than did the lines group $(\mathrm{M}=0.5, \boldsymbol{S D}=0.7)$. A 2 (condition) by $2(\mathrm{sex})$ ANOVA on the percentage of failure-to-compensate errors revealed only a main effect of condition, $F(1,20)=27.7, p<.001$.

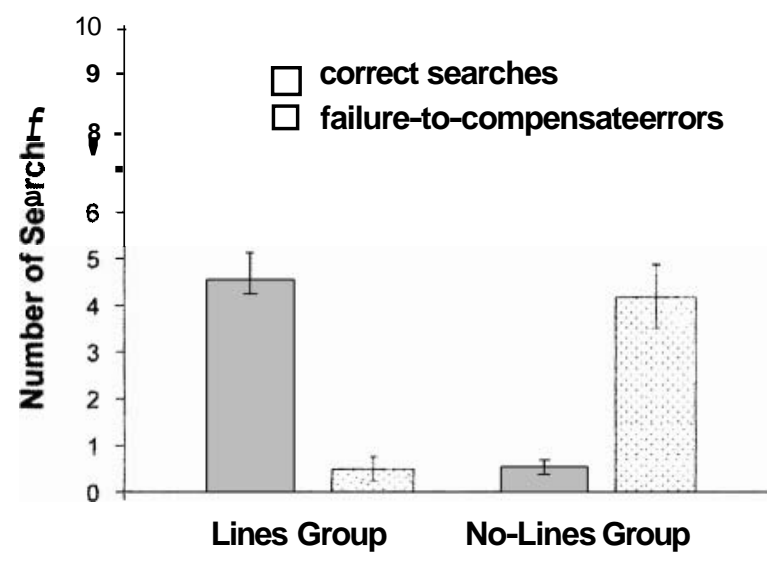

Figure 7. The distribution of correct and failure-to-compensatesearches in Study 3.

\section{Discussion}

As predicted, there were substantial differences in the performance of the two groups. The performance of the no-lines group, who did not see the dog pattern, essentially replicated that of children in prior studies (Blades et al., 1995; Presson, 1982; Pufall $\&$ Shaw, 1973). The no-lines group found the toy on only $5 \%$ of their searches, and they often made failure-to-compensate errors. In contrast, the lines group performed more than eight times better than the no-lines group and rarely made failure-to-compensate errors.

The results suggest that seeing the dog pattern provided a structure that helped the children compensate for the rotation of the map. More specifically, seeing the lines on the map could have helped children in two related ways. First, the lines could have emphasized the significance or relevance of the rotation for finding the toy. Seeing the dog in a noncanonical orientation on each trial may have reminded the children that the map was rotated. Even though children were told (and shown) during the orientation that the map was rotated, seeing the rotated dog may have made this information more salient and more accessible. Second, seeing the lines on the map could also have influenced how children established mappings between information on the map and information in the represented space. When the children entered the space to find the sticker, they could use the dog pattern to help them locate the toy. The systematic relation between the parts remained constant despite the rotation. The relations among locations in the dog thus would not have been tied to the egocentric viewpoint from which the no-lines group attempted to use the map. For example, if the children thought that the sticker was hidden in the face of the $\mathrm{dog}$, then they could look in the space for locations that were in or near the face. Consequently, they searched either at or relatively close to the target locations, whereas the no-lines group searched at locations that were $+90^{\circ}$ (or $-90^{\prime \prime}$ ) away from the correct locations.

These results differ substantially from those of previous studies involving the use of rotated maps. In most previous studies, children performed poorly, just as the no-lines group in the present study did. When children performed well in previous studies (e.g., Blades et at., 1995; Presson, 1982), they established mappings exclusively on the basis of salient, unique landmarks (e.g., windows or furniture) that were shown on the map and in the represented space. In contrast, in the present study, there were no distinctive landmarks present in the space that were also shown on the map. In addition, because there were no lines in the actual search space, parts of the dog became distinctive only if the children inferred that the locations in the room could form the outline of the dog. Moreover, they would have needed to map and use the spatial relations that defined the dog pattern. Therefore, this is the first study to demonstrate that young children can compensate for a rotation based on an inference regarding how the relations among information on the map correspond to relations among the locations. The systematicity of the pattern we used helped the children keep track of the sets of relations that defined the dog pattern even when these relations were not shown in a canonical orientation.

Finally, it is important to note that despite the relatively good performance of the lines group, their performance was worse than in Study 1, in which the map was correctly aligned with the space. 
The lines group in Study 4 clearly was able to compensate for the rotation. but they still faced the difficult task of discriminating among many different locations on the basis of a rotated representation of those locations. Nevertheless, despite their difficulty, they still performed much better than the no-lines group did and much better than children in most previous studies.

\section{General Discussion}

To use a map effectively, people must think about relations among the depictions of locations on maps and the corresponding referents in the world. Prior work has established that reasoning about such correspondences can be a challenging task for young children, particularly when they must take into account the spatial relations among multiple locations. The present studies substantially extend this prior work in two ways. First, the results shed light on why children have difficulty using spatial correspondences. Second, the results highlight the importance of systematicity as an organizing principle for establishing spatial correspondences and for thinking about relations more generally. The results thus contribute to an emerging line of research that highlights similarities between map reading and analogical reasoning.

We first consider how seeing the systematic dog pattern facilitated the 5-year-olds' performance. We then consider the implications of our findings and make suggestions for future research.

\section{How Did Seeing the Dog Pattern Facilitate Performance?}

Our claim throughout has been that seeing the dog pattern helped children think systematically about relations among sets of locations. To support this claim, we consider in this section whether alternate explanations can adequately account for the results. Specifically, we discuss and refute two possible accounts for our results that are derived, in part, from prior work on the development of map-reading skills. We then explain why the results indicate that the systematic pattern facilitated the use of spatial correspondences.

Were children thinking only of object correspondences? One possible explanation for the results is that the 5-year-olds who saw the dog pattern thought about locations in terms of isolated object correspondences. This explanation posits that the lines group performed well for the same reasons that children performed well in prior studies: They focused on unique, one-to-one correspondences, without mapping sets of spatial relations (see Blades \& Cooke, 1994).

For both conceptual and empirical reasons, the performance of the 5-year-olds in the lines group cannot be explained solely in terms of the use of object correspondences. In previous studies, object correspondences always involved either unique hiding locations or distinctive landmarks. Consequently, children could search correctly without mapping spatial relations. For example, the children could remember that the toy was hidden under the couch or next to a single window (Blades \& Cooke, 1994; Bluestein \& Acredolo, 1979; Lillard, 1993; Perner, 1991; Presson, 1982). In contrast, in the present studies, the locations were not unique. We asked children to find 1 location out of 27 alternatives, and all of the locations were identical except for spatial position.

One might argue instead that it was the parts of the dogs that provided the basis for establishing object correspondences. For example, a particular location could be coded as the nose or the tail. In this view, seeing the dog pattern allowed the children to think of locations as if they were individual objects within the dog. This explanation cannot fully account for our results, largely because the lines were shown only on the map, not in the space. The lines map, which showed the dog pattern and conveyed an advantage, actually looked less like the room than the no-lines map did. The children could not directly see the individual parts of the dog in the space, and hence they could not establish mappings using perceptually based object relations. Hence, the mechanism by which children could use the dog pattern to find the toy differs fundamentally from the use of object correspondences in previous studies.

In addition, the pattern of correct and incorrect searches differed from what we would expect if children were searching solely on the basis of object correspondences. Seeing the dog pattern conveyed the greatest advantage at locations that were not easily defined as single, distinct objects. For example, consider the location shown in the top panel of Figure 4. Most of the 5-year-olds in the lines group searched correctly when we hid the sticker here. In contrast, most of the 5-year-olds in the no-lines group searched at collinear locations proximal to the target. This target location could be construed as being in the belly of the dog. However, in the room, the belly location was not visually distinctive; it was simply one of several locations in line with other locations. Thus, a pure object-based correspondence would not be adequate to help children find the toy at this location. The belly could become distinctive in the room only if children first thought about how this one location was related to other locations, such as those in the legs. Put simply, the belly did not exist independently of the other parts, and the defining parts only existed in the room if the children used spatial correspondences.

A similar analysis applies to the location shown in the middle panel of Figure 4. The no-lines group tended to search (incorrectly) at locations that were roughly in line with the target; the no-lines group made fewer errors of this type. This location again is not easily construed as a single, visually distinctive part of the dog's body. It could perhaps be defined as the neck or the shoulders, but in this figure the neck and the shoulders do not exist independently of the head and the upper body. In addition, the data suggest that children may have used their knowledge of the dog to avoid the foils rather than simply to establish a one-to-one mapping. That is, the data suggest that the children may have attempted to avoid foil locations that were clearly in the head or in the trunk of the body. This strategy depends on the use of relations among multiple locations; it could not work if children only mapped object correspondences.

Finally, the developmental pattern in our results suggests that the benefit of seeing the dog pattern extended beyond helping children establish object correspondences. Four-year-olds did not benefit from seeing the dog pattern even though they performed much better than chance. Four-year-olds have performed well in previous mapping tasks that could be solved on the basis of object correspondences (e.g., Blades \& Cooke, 1994; DeLoache, 1989; Loewenstein \& Gentner, in press). Therefore, if seeing the dog pattern reduced the task to one that could be solved solely on the basis of object correspondences, then 4-year-olds in the lines group should have performed better than they did. 
Did seeing the dog pattern simply facilitate memory for the locations? A second possible explanation for the results is that seeing the dog pattern facilitated children's memory for the locations of the hidden objects. The children could augment information about spatial location (e.g., the target is to the left of several locations) with information about the location within the dog pattern (e.g., the target is in a paw). A strong version of this explanation is that seeing the dog only facilitated memory for the locations on the map because children only saw the dog pattern when they looked at the map. According to this view, the dog pattern helped children solely because it made the locations easier to remember, it did not affect how children mapped or interpreted the locations in the room.

Although seeing the dog pattern probably did help children encode the locations on the map, this effect cannot account fully for the results. The primary basis for this claim is again that the lines on the map were not present in the room. Children could encode the locations in terms of the dog pattern that they saw in the map, but for this information to be useful in the room, the children would need to map the dog pattern to the unlined locations in the room. Otherwise, they would face a mismatch between how the information was encoded and how it would need to be recalled. That is, children would have encoded the locations in terms of the dog pattern, but they would not be able to use the dog pattern to aid recall. Such a mismatch between how information was encoded (in terms of the dog) and how it would need to be recalled (without reference to the dog) seems unlikely to enhance memory given the numerous demonstrations of encoding specificity (e.g., Kulhavy, Stock, Peterson, \& Brooks, 1993; Tulving \& Thomson, 1973).

A weaker version of a memory-based explanation would posit that the dog pattern facilitated memory both at encoding (while studying the map) and at recall (while retrieving the sticker in the room). This explanation is at least in part consistent with our claim that children mapped the spatial relations that helped to define the dog pattern. If the dog pattern facilitated recall in the room, then the children must have been thinking that the coasters were related to each other and to the dog pattern. Thus, we do not deny that seeing the dog pattern on the map helped children remember locations, but we suggest that this facilitation required, a priori, that children map the dog pattern to the locations in the room.

In addition, the results of Study 4, in which the map was rotated relative to the represented space, strongly suggest that the effect we observed extends beyond memory facilitation. The two groups had very different search patterns. The lines group either found the sticker or searched close to the correct location, whereas the no-lines group failed to compensate for the rotation of the map. The errors of the no-lines group are difficult to explain on the basis of problems in remembering the location of the objects. Indeed, their errors indicate that they did remember what they saw on the map; a failure-to-compensate error requires, by definition, that children search at or very close to the location that would be correct if the map and space were correctly aligned. Where the problem arose was in translating what they remembered to the locations in the actual space.

Seeing the dog pattern facilitated relational mapping. For these reasons, we conclude that the effects of seeing the dog pattern on 5-year-olds' performance cannot be reduced solely to object mapping or the facilitation of encoding of the map-based information. The best explanation for the results is that the dog pattern helped the 5-year-olds to think about, remember, and map spatial relations. On the map, children could, as discussed above, use the dog pattern to help them encode the locations. Then, when they entered the room, the children could use the dog pattern to help them establish connections between relations on the map and the corresponding relations in the room. Making these mappings would, in turn, facilitate the recall of the correct location.

At each stage of this process, the systematicity of the dog pattern contributed to 5-year-olds' success. For example, knowing that a location was hidden in the belly could help the children to think about the relation between the legs and the lower body of the dog (see Figure 1). Thus, as in establishing an analogy, the children knew that a relation between a part and a whole that they saw on the map could be mapped to a relation that would exist in the room. Knowing these patterns of relations helped to highlight the correct location and to constrain possible searches at incorrect locations. Put simply, the dog pattern worked because it helped the 5-yearolds think about (a) how locations on the map were related to each other and (b) how these relations could be mapped to the unlined locations in the room.

It is important to note that we are not ruling out the possibility that seeing the dog pattern helped both to highlight individual locations and to make them more memorable. Indeed, the data strongly suggest that both did occur. We argue, however, that these effects are consequences of the use of the spatial correspondences that helped define the dog pattern. In this regard, our results are very different from those of prior studies, in which children could solve the search task without thinking about or using spatial correspondences.

\section{Implications and Questions for Future Research}

The findings, and our explanation for them, have important implications for research on the development of map-reading skills and spatial cognition. We consider three of these implications in this section.

Using spatial correspondences. The results highlight the unique importance of relational thinking for the development of map-reading skills. Children have difficulty using relational information to pinpoint the location at which to search. We provided a structure, the dog pattern, that made it easier for children to think about relations and that facilitated their performance substantially. The children were able to recreate, from memory, the relations among locations that formed the dog pattern.

Our explanation for the results has important implications for research on the process by which children use spatial correspondences. Specifically, we suggest that processes of remembering and mapping spatial correspondences are reciprocally related. In the present studies, encoding the locations on the map in terms of the dog pattern may have helped to remind the children that the locations in the room could be interpreted as forming a dog. The children then attempted to relate what they saw on the map to the unlined locations in the room. Finally, making these mappings facilitated the recall of the information they encoded from looking at the map. When children must keep track of and map several spatial locations, the processes of remembering and mapping the locations may be intertwined (Loewenstein, 2000).

Systematic patterns and spatial development. Our results imply that learning to take advantage of systematic patterns may be 
one important aspect of the development of map-reading skills. Adults often use familiar, systematic patterns to make spatial information easier to remember and to map. For example, describing a location in terms of the "panhandle" of Texas makes specific locations easier to communicate and easier to locate on a map. In this example, people take advantage of the systematic relation between pans and their handles to remember and map spatial relations in the real world. The present studies are the first to investigate specifically the development of the ability to use systematic spatial patterns to facilitate mapping and search.

Our results raise several questions for future research regarding the development of children's use of spatial correspondences. For example, one question concerns whether younger children could benefit from a systematic pattern. We found that only 5 -year-olds could benefit from seeing the dog pattern. In this regard, our results are similar to those of other researchers who have examined children's use of higher order patterns in other spatial tasks. For example, Johnson (1999) found that children younger than 5 years old have difficulty using a picture of a completed puzzle as a guide for assembling individual pieces. These and other results suggest that the development of the ability to think systematically about the relation between parts and whole may be a critical achievement of children between the ages of 4 and 5 years.

However, it is also possible that the task, the number of locations, or the pattern of locations that we used was simply too complicated for 3- and 4-year-olds and that these children could have benefited from seeing a simpler pattern. Perhaps, for example, 3- and 4-year-olds could gain an advantage from seeing only the locations that formed the face of the dog. Success in a simpler task might provide a basis for transfer of the acquired knowledge to a more difficult task, such as the ones we used here. The facilitative effect of transfer has been demonstrated in previous studies of spatial cognition and the use of map and models as well as in other domains of cognitive development (e.g., Brown, Kane, \& Long, 1989; Kotovsky \& Gentner, 1996; Marzolf \& DeLoache, 1994; Pick, Pick, \& Thomas, 1966; Uttal, Schreiber. \& DeLoache, 1995).

A second question concerns the development of the ability to use systematic patterns spontaneously. In the present studies, children performed well only when we connected the locations with lines; hearing a label without seeing the lines (Study 3) conveyed no advantage. Future studies could investigate when, and how, children can (a) benefit from labels and (b) look for and find structure without either a visual example or a label. Part of this development may involve acquiring knowledge of an increasingly large number of figures, but it may also involve learning to search actively for ways of organizing spatial information (see Axia \& Caravaggi, 1987; Mandler \& Parker, 1976; Stiles et al., 1990; Vurpillot, 1976). In this regard, it is interesting to note that the performance of 2 five-year-olds in the no-lines group in Study I was as good as the average level of performance in the lines group and that 1 of them spontaneously said that the structure formed a dog pattern.

A third question concerns the development of the ability to apply systematic patterns to real-world spaces. Our developmental analysis implies that children eventually learn to apply systematic $\mathrm{p}^{\text {atterns }}$ to spaces of geographic scale (e.g., locations in a city or state). Thus it will be important in future studies to extend the work to larger and more realistic spaces. For example, search locations could be distributed across the grounds of a school or even across parts of a city (e.g., Cornell, Heth, \& Alberts, 1994; Heth, Cornell, \& Alberts, 1997).

Maps are like analogies. More generally, the results also contribute to a growing body of literature that highlights important similarities between the use of maps and analogical reasoning. We conceived of map reading as a form of analogy. Consequently, we construed the problems that children typically have in using spatial correspondences as symptomatic of the general difficulty that they have in reasoning about relational similarity (Gentner \& Ratterman, 1991). This led us to predict that a systematic pattern might facilitate children's use of spatial correspondences both by highlighting relations among locations and by helping children to notice correspondences between relations on the map and in the space. Researchers are now investigating how other techniques, also adapted from analogy research, can facilitate children's understanding and use of maps. For example, having children compare sets of relations in two models facilitates children's use of spatial correspondences (Loewenstein \& Gentner, in press), and language that specifically highlights spatial relations (i.e., provides more than a simple verbal label) does facilitate the use of spatial correspondences (Loewenstein, 2000; Loewenstein \& Gentner, 1998).

In conclusion, the findings of these studies extend our understanding of the development of spatial cognition and map reading in two related ways. First, we have provided evidence for the similarity of the cognitive processes involved in using maps and establishing analogies. In both cases, children face the challenge of thinking about how sets of relations can be mapped to different objects or locations. Young children typically have difficulty using the spatial relations on maps because they tend to focus on superficial, perceptual similarities between the depictions of individual locations on the maps and the corresponding individual locations in the space. As in analogical reasoning, they have trouble taking relational information into account. Second, our results indicate that 5-year-olds' use of spatial correspondences can be enhanced substantially by providing a framework that makes relations more accessible and tractable. Our results are thus the first to highlight possible mechanisms by which young children may begin to move beyond the limitations that keep them from taking full advantage of the spatial information that maps can provide.

\section{References}

Anderson, J. R. (1995).Cognitivepsychology and its implications (4thed.). New York: Freeman.

Axia, G., \& Caravaggi,D. (1987). Effects of spatial arrangement on 4 and 6-year-old children's memory. Perceptual and Motor Skills, 65, 283293.

Bence, V. M., \& Presson, C. C. (1997, April). The spatial basis of young children's use of scale models. Paper presented at the 1997 Biennial Meeting of the Society for Research in Child Development, Washington, DC.

Biederman, I. (1987). Recognition-by-components: A theory of human image understanding. Psychological Review, 94, 115-147.

Blades, M., \& Cooke, Z. (1994). Young children's ability to understand a model as a spatial representation. Journal of Genetic Psychology, 155, 201-218.

Blades, M., Sowden, S., \& Spencer, C. (1995). Young children's use of spatial relationships in tasks with maps and models, Cartographica. 32, $18-29$. 
Blades, M., \& Spencer, C. (1987). The use of maps by 4-6-yr-old children in a large-scale maze. British Journal of Developmental Psychology, 5, $19-24$.

Blades, M., \& Spencer, C. (1994). The development of children's ability to use spatial representations. In H. W. Reese (Ed.), Advances in child development and behavior (Vol. 25, pp. 157-199). San Diego, CA: Academic Press.

Bluestein, M., \& Acredolo, L. (1979). Developmental changes in map reading skills. Child Development, 50, 691-697.

Boltyanskii, V. G., \& Gohberg, I. T. (1980). The decomposition offigures into smaller parts (H. Christoffers \& T. P. Branson, Trans.). Chicago: University of Chicago Press.

Bremner, J. G., \& Andreasen, G. (1998). Young children's ability to use maps to find ways in novel spaces. British Journal of Developmental Psychology, 16, 197-218

Brown, A. L., Kane, M. J., \&Long, C. (1989). Analogical transfer in young children: Analogies as tools for communication and exposition. Applied Cognitive Psychology, 3, 275-293.

Corballis, M. C., \& Beale, I. L. (1976). The psychology of left and right. Hillsdale, NJ: Erlbaum.

Comell, E. H., Heth, C. D., \& Alberts, D. M. (1994). Place recognition and way finding by children and adults. Memory \& Cognition, 22, 633-643.

DeLoache, J. S. (1987). Rapid change in the symbolic functioning of very young children. Science, 238, 1556-1557.

DeLoache, J. S. (1989). Young children's understanding of the correspondence between a scale model and a larger space. Cognitive Development, 4, 121-139.

DeLoache, J. S. (2000). Dual representation and young children's use of scale models. Child Development, 71, 329-338.

Dow, G. A., \& Pick, H. L. (1992). Young children's use of models and photographs as spatial representations. Cognitive Development, 7, 351363.

Gentner, D. (1977). Children's performance on a spatial analogies task. Child Development, 48, 1034-1039.

Gentner, D., \& Ratterman, M. J. (1991). Language and the career of similarity. In S. A. Gelman \& J. P. Byrnes (Eds.), Perspectives on language and thought: Interrelations in development (pp. 225-277). London: Cambridge University Press.

Gentner, D., \& Toupin, C. (1986). Systematicity and surface similarity in the development of analogy. Cognitive Science, 10, 277-300.

Heth, D. C., Comell, E. H., \& Alberts, D. M. (1997). Differential use of landmarks by 8 - and 12-year-old children during route reversal navigation. Journal of Environmental Psychology, 17, 199-213.

Hoffman, D. D., \& Richards, W. A. (1984). Parts of recognition. Cognition, 18, 65-96.

Hoffman, D. D., \& Singh, M. (1997). Salience of visual parts. Cognition, 63, 29-78.

Hunt, E., \& Agnoli, F. (1991). The Whorfian hypothesis: A cognitive psychology perspective. Psychological Review, 98, 377-389.

Huttenlocher, J., \& Presson, C. C. (1973). Mental rotation and the perspective problem. Cognitive Psychology, 4, 277-299.

Huttenlocher, J., \& Presson, C. C. (1979). The coding and transformation of spatial information. Cognitive Psychology, 11, 375-394.

Johnson, K. S. (1999, April). Missing the big picture: How preschoolers assemblejigsaw puzzles. Paper presented at the Biennial Meetings of the Society for Research in Child Development, Albuquerque, NM.

Koffka, K. (1963). Principles of gestalt psychology. New York: HarcourtBrace.

Kohler, W. (1947). Gestaltpsychology: An introduction to new concepts in modern psychology. New York: Liveright.

Kotovsky, L., \& Gentner, D. (1996). Comparison and categorization in the development of relational similarity. Child Development, 67, 27972822.

Kulhavy, R., Stock, W., Peterson, S. E., \& Brooks, R. (1993). Encoding specificity: The case of maps and text. Bulletin of the Psychonomic Society, 31, 128-130.

Landau, B. (1986). Early map use as an unlearned ability. Cognition, 22, 201-223.

Levine, M., Jankovic, I. N., \& Palij, M. (1982). Principles of spatial problem solving. Journal of Experimental Psychology: General, 111, $157-175$.

Liben, L. S. (1999). Developing an understanding of external spatial representations. In I. E. Sigel (Ed.), Development of representational thought: Theories and applications (pp. 297-321). Hillsdale, NJ: Erlbaum.

Liben, L. S., \& Downs, R. (1989). Understanding maps as symbols: The development of map concepts in children. In H. W. Reese (Ed.), $A d$ vances in child development and behavior (Vol. 22, pp. 146-202). San Diego, CA: Academic Press.

Liben, L. S., \& Downs, R. M. (1993). Understanding person-space-map relations: Cartographic and developmental perspectives. Developmental Psychology, 29, 739-752.

Liben, L. S., \& Yekel, C. A. (1996). Preschoolers' understanding of plan and oblique maps: The role of geometric and representational correspondence. Child Development, 67, 2780-2796.

Lillard, A. S. (1993). Pretend play skills and the child's theory of mind. Child Development, 64, 348-371.

Loewenstein, J. (2000). Relational mapping in preschoolers. Unpublished doctoral dissertation, Northwestern University.

Loewenstein, J., \& Gentner, D. (1998). Relational language facilitates analogy in children. In Proceedings of the Twentieth Annual Conference of the Cognitive Science Society (p. 1219). Mahwah, NJ: Erlbaum.

Loewenstein, J., \& Gentner, D. (in press). Spatial mapping in preschoolers: Close comparisons facilitate far mappings. Journal of Cognition and Development.

Mandler, J. M., \& Parker, R. E. (1976). Memory for descriptive and spatial information in complex pictures. Journal of Experimental Psychology: Human Learning and Memory, 2, 38-48.

Marzolf, D. P., \& DeLoache, J. S. (1994). Transfer in young children's understanding of spatial representations. Child Development, 65, 1-15.

Marzolf, D. P., DeLoache, J. S., \& Kolstad, V. (1999). The role of relational similarity in young children's use of a scale model. Developmental Science, 2, 296-305.

Morrison, J. B., \& Tversky, B. (1997). Body schemas. In Proceedings of the meetings of the Cognitive Science Society (pp. 525-529). Mahwah, NJ: Erlbaum.

Olson, D. R., \& Bialystock, E. (1983). Spatial cognition. Hillsdale, NJ: Erlbaum.

Palmer, S. (1975). The effects of contextual scenes on the identification of objects. Memory \& Cognition. 3, 519-526.

Pemer, J. (1991). Understanding the representational mind. Cambridge, MA: MIT Press.

Pick, A. D., Pick, H. L., \& Thomas, M. L. (1966). Cross-modal transfer and improvement of form discrimination. Journal of Experimental Child Psychology, 3, 279-288.

Presson, C. C. (1982). The development of map-reading skills. Child Development, 53, 196-199.

Pufall, P. B., \& Shaw, R. E. (1973). Analysis of the development of children's spatial reference systems. Cognitive Psychology, 5, 151-175.

Schoenfeld, A. H., \& Henmann, D. J. (1982). Problem perception and knowledge structure in expert and novice mathematical problem solvers. Journal of Experimental Psychology: Learning, Memory, and Cognition, 8, 484-494.

Silver, E. A. (1981). Recall of mathematical problem information: Solving related problems. Journal of Research in Mathematics Education, 24, 117-135.

Smart, J. R. (1986). Modem geometries (3rd ed.). Pacific Grove, CA: Brooks/Cole. 
Stiles, J., Delis, D. C., \& Tada, W. L. (1991). Global-local processing in preschool children. Child Development, 62, 1258-1275.

Stiles, J., Tada, W., \& Whipple, T. (1990). Facilitative effects of labeling on preschool children's copying of simple geometric forms. Perceptual \& Motor Skills. 70, 663-672.

Tarr, M. J., \& Pinker, S. (1989). Mental rotation and orientationdependence in shape recognition. Cognitive Psychology, 21, 233-282.

Tulving, E., \& Thomson, D. M. (1973). Encoding specificity and retrieval processes in episodic memory. Psychological Review, 80, 359-380.

Tversky, B. (1989). Parts, partonomies, and taxonomies. Developmental Psychology, 25, 983-995.

Tversky, B. (1997, September). Three spaces of spatial cognition. Paper presented at the Workshop on Spatial Cognition, Rome, Italy.

Tversky, B., \& Hemenway, K. (1984). Objects, parts, and categories. Journal of Experimental Psychology: General, 113, 169-193.

Uttal, D. H. (1996). Angles and distances: Children's and adults' reconstruction and scaling of spatial configurations. Child Development, 67, 2763-2779.
Uttal, D. H. (2000). Seeing the big picture: Map use and the development of spatial cognition. Developmental Science, 3, 247-264.

Uttal, D. H., Loewenstein, J., \& Gentner, D. (2000, May). On the relation between maps and analogies. Paper presented at the Conference on Conceptual Structure, Language, and Discourse, Santa Barbara, CA.

Uttal, D. H., Schreiber, J. C., \& DeLoache, J.S. (1995). Waiting to use a symbol: The effects of delay on children's use of models. Child Development, 66, 1875-1889.

Uttal, D. H., \& Wellman, H. M. (1989). Young children's representation of spatial information acquired from maps. Developmental Psychology, 25, 128-138.

Vurpillot, E. (1976). The visual world of the child. (W. E. C. Gillham, Trans.). London: Allen \& Unwin.

Wood, D. (1992). The power of maps. New York: Guilford Press.

Received January 28, 1998 Revision received December 6, 2000 Accepted December 15, 2000 